\title{
Two-loop leading-color helicity amplitudes for three-photon production at the LHC
}

\author{
Herschel A. Chawdhry, ${ }^{a}$ Michał Czakon, ${ }^{b}$ Alexander Mitov ${ }^{c}$ and Rene Poncelet ${ }^{c}$ \\ ${ }^{a}$ Rudolf Peierls Centre for Theoretical Physics, Clarendon Laboratory, University of Oxford, \\ Park Road, Oxford OX1 3PU, U.K. \\ ${ }^{b}$ Institut für Theoretische Teilchenphysik und Kosmologie, RWTH Aachen University, \\ Sommerfeldstr. 16, D-52056 Aachen, Germany \\ ${ }^{c}$ Cavendish Laboratory, University of Cambridge, \\ J.J. Thomson Avenue, Cambridge CB3 OHE, U.K. \\ E-mail: herschel.chawdhry@physics.ox.ac.uk, \\ mczakon@physik.rwth-aachen.de, adm74@cam.ac.uk, \\ poncelet@hep.phy.cam.ac.uk
}

AbStRaCt: We calculate all planar contributions to the two-loop massless helicity amplitudes for the process $q \bar{q} \rightarrow \gamma \gamma \gamma$. The results are presented in fully analytic form in terms of the functional basis proposed recently by Chicherin and Sotnikov. With this publication we provide the two-loop contributions already used by us in the NNLO QCD calculation of the LHC process $p p \rightarrow \gamma \gamma \gamma$ [Chawdhry et al. (2019)]. Our results agree with a recent calculation of the same amplitude [Abreu et al. (2020)] which was performed using different techniques. We combine several modern computational techniques, notably, analytic solutions for the IBP identities, finite-field reconstruction techniques as well as the recent approach [Chen (2019)] for efficiently projecting helicity amplitudes. Our framework appears well-suited for the calculation of two-loop multileg amplitudes for which complete sets of master integrals exist.

KEYwords: Perturbative QCD, Scattering Amplitudes

ARXiv EPrint: 2012.13553 


\section{Contents}

1 Introduction 1

2 Computation of the helicity amplitudes $\quad 2$

2.1 Notation and renormalization 2

2.2 Helicity projections 4

$\begin{array}{lll}2.3 & \text { Reduction to pentagon functions } & 7\end{array}$

$\begin{array}{lll}3 & \text { Results } & 11\end{array}$

4 Conclusion $\quad 13$

A Renormalization constants 13

\section{Introduction}

Tree-level and multi-loop amplitudes are the building blocks of predictions of gauge field theories and, in particular, for observables in high-energy scattering experiments. For this reason a lot of theoretical effort has been invested in their evaluation and in the development of ever-more efficient methods for their calculation. A number of gauge theories are being studied, starting from string and supersymmetric theories [1-13], to gravity [14-16], to Yang-Mills [17, 18], to QED [19] and QCD [20-32] and ultimately to the full Standard Model [33-42]. Research on generic methods for solving multi-loop integrals is also ongoing [43-61].

In this work we focus our attention on QCD which is the theory most relevant to high-precision physics at the Large Hadron Collider. Within QCD, the current frontier for $2 \rightarrow 1$ and $2 \rightarrow 2$ processes is three loops [62-68], while for $2 \rightarrow 3$ scattering processes with massless partons it is two loops. A lot of work has already been carried out in this direction, mostly for planar amplitudes [69-73] but recently also for non-planar ones $[49,57,59,74-77]$. All this progress has enabled the very recent first calculation of a $2 \rightarrow 3$ process at NNLO, namely, three-photon production at the LHC. This has been achieved by two separate groups [33, 42] following very different methods. An essential ingredient for these calculations was the corresponding two-loop amplitude $q \bar{q} \rightarrow \gamma \gamma \gamma$. Both references have evaluated it in the leading-color approximation, following different computational approaches. The actual results for the amplitude have, until very recently, not been publicly available.

The goal of the present work is to complete this gap and present the explicit analytic result for the leading-color two-loop amplitude $q \bar{q} \rightarrow \gamma \gamma \gamma$ used in the NNLO calculation of ref. [33]. We also compare our result with the one used in ref. [42] and published recently 
in ref. [78]. We find full agreement between the two results. Our subsequent discussion will be focused on methods used for the evaluation of the amplitude and we refer the interested reader to ref. [33] for a broader introduction to the problem and the subject, the definition of the leading color approximation for this process as well as the implications of this calculation.

The paper is organized as follows. In section 2 we detail the evaluation of the amplitude. Specifically, in section 2.1 we introduce our notation and define the finite remainder; in section 2.2 we describe the method for projecting helicities, while in section 2.3 we explain how the rational coefficients of the amplitude are derived. Our results are presented in section 3. They are available for download in electronic form with the arXiv submission of this work.

\section{Computation of the helicity amplitudes}

\subsection{Notation and renormalization}

We consider the partonic process

$$
q_{c}^{h_{1}}\left(p_{1}\right) \bar{q}_{c^{\prime}}^{h_{2}}\left(p_{2}\right) \rightarrow \gamma^{h_{3}}\left(p_{3}\right) \gamma^{h_{4}}\left(p_{4}\right) \gamma^{h_{5}}\left(p_{5}\right),
$$

where $h_{i} \in\{+,-\}$ denotes the helicity of the $i$ th parton, $i=1, \ldots, 5$. The indices $c, c^{\prime}$ denote quarks' color. All partons are massless and on-shell $p_{i}^{2}=0$. Momentum conservation and on-shell conditions leave five independent parity-even Lorentz invariants $s_{i j}=\left(p_{i}+p_{j}\right)^{2}$ and one parity-odd $\operatorname{tr}_{5}=4 i \varepsilon_{p_{1} p_{2} p_{3} p_{4}}$. We choose the following set of variables to parameterize the amplitudes

$$
x=\left\{s_{12}, s_{23}, s_{34}, s_{45}, s_{51}, \operatorname{tr}_{5}\right\} .
$$

All other Lorentz invariants can be expressed in terms of this set:

$$
\begin{aligned}
& s_{13}=s_{12}-s_{23}-s_{45} \\
& s_{14}=-s_{15}+s_{23}+s_{45} \\
& s_{24}=s_{15}-s_{23}+s_{34} \\
& s_{25}=s_{12}-s_{15}-s_{34} \\
& s_{35}=s_{12}-s_{34}-s_{45} .
\end{aligned}
$$

The physical scattering region satisfies [47]

$$
s_{12}>0, \quad s_{12} \geq s_{34}, \quad s_{45} \leq s_{12}-s_{34}, \quad s_{23}>s_{12}-s_{45}, \quad s_{51}^{-} \leq s_{51} \leq s_{51}^{+}, \quad\left(\operatorname{tr}_{5}\right)^{2}<0,
$$

with

$$
\begin{aligned}
\left(\operatorname{tr}_{5}\right)^{2}= & s_{12}^{2}\left(s_{23}-s_{51}\right)^{2}+\left(s_{23} s_{34}+s_{45}\left(s_{34}+s_{51}\right)\right)^{2}- \\
& 2 s_{12}\left(s_{23}^{2} s_{34}+s_{23} s_{34} s_{45}-s_{23}\left(s_{34}+s_{45}\right) s_{51}+s_{45} s_{51}\left(s_{34}+s_{51}\right)\right),
\end{aligned}
$$


and

$$
\begin{aligned}
s_{51}^{ \pm}= & \frac{1}{\left(s_{12}-s_{45}\right)^{2}}\left(s_{12}^{2} s_{23}+s_{12} s_{34} s_{45}-s_{23} s_{34} s_{45}-s_{34} s_{45}^{2}-\right. \\
& \left.s_{12} s_{23}\left(s_{34}+s_{45}\right) \pm 2 \sqrt{s_{12} s_{23} s_{34} s_{45}\left(s_{45}+s_{23}-s_{12}\right)\left(s_{45}+s_{34}-s_{12}\right)}\right) .
\end{aligned}
$$

The UV renormalized amplitude for this process is denoted by

$$
\mathcal{M}\left(\alpha_{s}\right)_{c c^{\prime}}^{h_{1} h_{2} h_{3} h_{4} h_{5}}(x)=\delta_{c c^{\prime}} \mathcal{M}\left(\alpha_{s}\right)^{h_{1} h_{2} h_{3} h_{4} h_{5}}(x) \equiv \delta_{c c^{\prime}} \mathcal{M}^{\bar{h}}
$$

where we factored out the (trivial) color dependence. We summarize the helicity configuration by $\bar{h}=\left\{h_{1}, h_{2}, h_{3}, h_{4}, h_{5}\right\}$ and suppress the kinematic dependence for brevity. The amplitude can be expanded in $\alpha_{s}$

$$
\mathcal{M}^{\bar{h}}=\mathcal{M}^{\bar{h}(0)}+\left(\frac{\alpha_{s}}{4 \pi}\right) \mathcal{M}^{\bar{h}(1)}+\left(\frac{\alpha_{s}}{4 \pi}\right)^{2} \mathcal{M}^{\bar{h}(2)}+\mathcal{O}\left(\alpha_{s}^{3}\right) .
$$

The UV renormalized amplitude $\mathcal{M}^{\bar{h}}$ is related to the bare amplitude computed in $d=4-2 \varepsilon$ dimensions $\mathcal{M}^{\bar{h}, B}$ through

$$
\mathcal{M}^{\bar{h}}\left(\alpha_{s}\right)=\left(\frac{\mu^{2} e^{\gamma_{E}}}{4 \pi}\right)^{-2 \varepsilon} Z_{q} \mathcal{M}^{\bar{h}, B}\left(\alpha_{s}^{0}\right),
$$

where $Z_{q}$ is the light quark wave-function renormalization constant. The bare coupling $\alpha_{s}^{0}$ is renormalized in the $\overline{\mathrm{MS}}$ scheme according to

$$
\alpha_{s}^{0}=\left(\frac{e^{\gamma_{E}}}{4 \pi}\right)^{\varepsilon} \mu^{2 \varepsilon} Z_{\alpha_{s}} \alpha_{s} .
$$

All UV renormalization constants are given in appendix A.

The IR divergences of the UV renormalized amplitude can be factorized by means of the so-called $\mathbf{Z}$ operator:

$$
\mathcal{M}^{\bar{h}}=\mathbf{Z} \mathcal{F}^{\bar{h}} .
$$

Once the $\mathbf{Z}$-factor, the finite-remainder $\mathcal{F}$ and the amplitude $\mathcal{M}$ have been expanded in powers of $\alpha_{s} /(4 \pi)$, eq. (2.15) reduces to

$$
\begin{aligned}
& \mathcal{M}^{\bar{h}(0)}=\mathcal{F}^{\bar{h}(0)} \\
& \mathcal{M}^{\bar{h}(1)}=\mathbf{Z}^{(1)} \mathcal{M}^{\bar{h}(0)}+\mathcal{F}^{h(1)}, \\
& \mathcal{M}^{\bar{h}(2)}=\mathbf{Z}^{(2)} \mathcal{M}^{\bar{h}(0)}+\mathbf{Z}^{(1)} \mathcal{F}^{\bar{h}(1)}+\mathcal{F}^{\bar{h}(2)} .
\end{aligned}
$$

We define $\mathbf{Z}$ in the $\overline{\mathrm{MS}}$ scheme. This completely specifies the finite remainder $\mathcal{F}^{\bar{h}}$. The explicit expansion for $\mathbf{Z}$ through two-loops in QCD is given in appendix A.

The amplitude can be decomposed in color and electric-charge structures. For the two-loop finite remainder we find four non-vanishing contributions

$$
\begin{aligned}
\mathcal{F}^{\bar{h}(2)}(q \bar{q} \rightarrow \gamma \gamma \gamma)= & Q_{q}^{3}\left(4 C_{F}^{2} \mathcal{F}^{\bar{h}, C_{F}^{2}}+4 C_{F} C_{A} \mathcal{F}^{\bar{h}, C_{F} C_{A}}+2 C_{F} n_{l} \mathcal{F}^{\bar{h}, C_{F} n_{f}}\right) \\
& +Q_{q}\left(\sum_{q^{\prime}} Q_{q^{\prime}}^{2}\right) 2 C_{F} \mathcal{F}^{\bar{h}, Q} .
\end{aligned}
$$



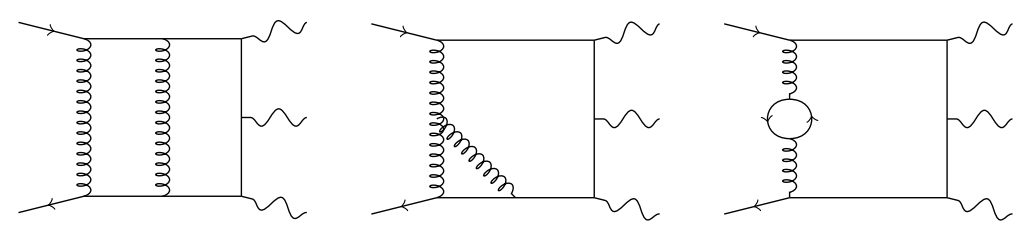

$\sim C_{F}^{2}$

$\sim C_{F} C_{A}$

$\sim C_{F} n_{f}$
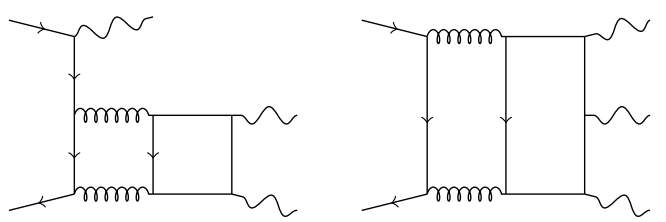

$$
\sim C_{F} \sum_{q^{\prime}} Q_{q}^{\prime 2} \quad \sim C_{F} \sum_{q^{\prime}} Q_{q}^{\prime 3}
$$

Figure 1. Representative two-loop diagrams and their color/charge factors.

In the above equation, $Q_{i}$ are quarks' QED couplings $\left(Q_{u, c, t}=\frac{2}{3} e\right.$ and $\left.Q_{d, s, b}=-\frac{1}{3} e\right)$. The sum over $q^{\prime}$ in eq. (2.19) goes over all massless quarks. The difference between the QED couplings in the first and second lines of eq. (2.19) is due to the following: all diagrams contributing to the first line of eq. (2.19) have all three photons coupling to the external quark line (of flavor $q$ ) while the diagrams contributing to the second line of eq. (2.19) have one photon coupling directly to the external quark line (of flavor $q$ ) and two photons coupling to an internal fermion loop with a flavor $q^{\prime}$. Example diagrams for the different contributions can be found in figure 1. In this work we do not consider diagrams with massive quark loops. We also note that the contribution proportional to $C_{F} \sum_{q^{\prime}} Q_{q^{\prime}}^{3}$ from diagrams with three photons coupling to an internal fermion loop (see figure 1) vanishes by Furry's theorem.

The leading color contribution of $\mathcal{F}^{\bar{h}(2)}(q \bar{q} \rightarrow \gamma \gamma \gamma)$ is proportional to $N_{c}^{2}$ and, as follows from eq. (2.19), is only dependent on a linear combination of the first two factors in the first line of eq. (2.19):

$$
\left.\mathcal{F}^{\bar{h}(2)}(q \bar{q} \rightarrow \gamma \gamma \gamma)\right|_{\text {l.c. }}=Q_{q}^{3} N_{c}^{2}\left(\mathcal{F}^{\bar{h}, C_{F}^{2}}+2 \mathcal{F}^{\bar{h}, C_{F} C_{A}}\right)+\mathcal{O}\left(N_{c}\right) .
$$

The phenomenological analysis in ref. [33] is based on eq. (2.20). The justification for the use of this approximation can be found in that reference.

Despite recent progress, the non-planar diagrams in this process are still beyond reach. For this reason, in this work we derive and present in analytic form the planar results for the following two factors:

$$
\mathcal{F}^{\bar{h}, C_{F}^{2}}+2 \mathcal{F}^{\bar{h}, C_{F} C_{A}} \text { and } \mathcal{F}^{\bar{h}, C_{F} n_{f}}
$$

\subsection{Helicity projections}

The process (2.1) has two independent helicity amplitudes. As such we choose

$$
\mathcal{F}^{\{+----\}} \equiv \mathcal{F}^{\bar{h}_{-}} \quad \text { and } \quad \mathcal{F}^{\{+---+\}} \equiv \mathcal{F}^{\bar{h}_{+}} .
$$


All other helicities can be obtained from this set by conjugation and/or permutation of external momenta. Since at tree-level $\mathcal{F}^{(0), \bar{h}_{-}}=0$, the one-loop contribution to this helicity is finite and its two-loop correction does not contribute to the squared matrix element for this process through two loops.

In order to extract the helicity amplitudes (2.22) in the 't Hooft-Veltman scheme we employ the projection method proposed in ref. [79]. Similar approaches have been advocated in refs. [80, 81]. The essence of this method is that, for a given helicity, it provides explicit prescription for constructing the external wave-functions. For the process under consideration, these wave-functions can be factored out by writing the amplitude in the following way

$$
\mathcal{M}^{\bar{h}}=\varepsilon_{3, h_{3}}^{* \mu} \varepsilon_{4, h_{4}}^{* \nu} \varepsilon_{5, h_{5}}^{* \rho} \bar{v}\left(h_{2}\right) \Gamma_{\mu \nu \rho} u\left(h_{1}\right) .
$$

The construction of the external wave-functions is done in 4 dimensions. On one hand this significantly simplifies the construction of a basis of polarization vectors. On the other, it introduces scheme dependence into the bare loop amplitude. As demonstrated in refs. $[79,82,83]$, however, this scheme dependence does not affect the finite remainder of the amplitude in the limit $\varepsilon \rightarrow 0$.

Our construction of the fermionic wave functions introduces the matrix $\gamma_{5}$. The fact that wave functions are constructed in 4 dimensions implies that we only use 4-dimensional identities for $\gamma_{5}$. With the help of these identities we eliminate all occurrences of $\gamma_{5}$ and, in this process, trade them for objects involving $\varepsilon^{\mu \nu \rho \sigma}$. Multiplying (and dividing) the term proportional to $\varepsilon^{\mu \nu \rho \sigma}$ by $\left(\operatorname{tr}_{5}\right)^{2}$ and recalling that $\left(\operatorname{tr}_{5}\right)^{2}$ is a polynomial function of the invariants $s_{i j}$ (see eq. (2.9)), we eliminate all occurrences of $\varepsilon^{\mu \nu \rho \sigma}$ using

$$
\begin{aligned}
\varepsilon_{p_{1} p_{2} p_{3} p_{4}} \varepsilon^{\mu \nu \rho \sigma}= & +p_{1}^{\rho} p_{2}^{\nu} p_{3}^{\mu} p_{4}^{\sigma}-p_{1}^{\nu} p_{2}^{\rho} p_{3}^{\mu} p_{4}^{\sigma}-p_{1}^{\rho} p_{2}^{\mu} p_{3}^{\nu} p_{4}^{\sigma}+p_{1}^{\mu} p_{2}^{\rho} p_{3}^{\nu} p_{4}^{\sigma}+p_{1}^{\nu} p_{2}^{\mu} p_{3}^{\rho} p_{4}^{\sigma}-p_{1}^{\mu} p_{2}^{\nu} p_{3}^{\rho} p_{4}^{\sigma} \\
& -p_{1}^{\rho} p_{2}^{\nu} p_{3}^{\sigma} p_{4}^{\mu}+p_{1}^{\nu} p_{2}^{\rho} p_{3}^{\sigma} p_{4}^{\mu}+p_{1}^{\rho} p_{2}^{\sigma} p_{3}^{\nu} p_{4}^{\mu}-p_{1}^{\sigma} p_{2}^{\rho} p_{3}^{\nu} p_{4}^{\mu}-p_{1}^{\nu} p_{2}^{\sigma} p_{3}^{\rho} p_{4}^{\mu}+p_{1}^{\sigma} p_{2}^{\nu} p_{3}^{\rho} p_{4}^{\mu} \\
& +p_{1}^{\rho} p_{2}^{\mu} p_{3}^{\sigma} p_{4}^{\nu}-p_{1}^{\mu} p_{2}^{\rho} p_{3}^{\sigma} p_{4}^{\nu}-p_{1}^{\rho} p_{2}^{\sigma} p_{3}^{\mu} p_{4}^{\nu}+p_{1}^{\sigma} p_{2}^{\rho} p_{3}^{\mu} p_{4}^{\nu}+p_{1}^{\mu} p_{2}^{\sigma} p_{3}^{\rho} p_{4}^{\nu}-p_{1}^{\sigma} p_{2}^{\mu} p_{3}^{\rho} p_{4}^{\nu} \\
& -p_{1}^{\nu} p_{2}^{\mu} p_{3}^{\sigma} p_{4}^{\rho}+p_{1}^{\mu} p_{2}^{\nu} p_{3}^{\sigma} p_{4}^{\rho}+p_{1}^{\nu} p_{2}^{\sigma} p_{3}^{\mu} p_{4}^{\rho}-p_{1}^{\sigma} p_{2}^{\nu} p_{3}^{\mu} p_{4}^{\rho}-p_{1}^{\mu} p_{2}^{\sigma} p_{3}^{\nu} p_{4}^{\rho}+p_{1}^{\sigma} p_{2}^{\mu} p_{3}^{\nu} p_{4}^{\rho},
\end{aligned}
$$

and then promoting all indices to $d$ dimensions. Once this has been achieved, the subsequent contractions of external polarization states with the rest of the amplitude are performed in $d$ dimensions. The remaining factor $\varepsilon_{p_{1} p_{2} p_{3} p_{4}}$ is converted into $\operatorname{tr}_{5}$ which is then treated as an independent kinematic variable that is included in the rational coefficients $A_{b}$ defined in section 2.3 below.

We next derive explicit expressions for the wave functions of the final state vector particles of given helicity $h= \pm 1$

$$
\varepsilon_{i, h}^{\mu} \quad \text { with } \quad i \in\{3,4,5\} .
$$

As a first step we replace those with new linear polarization vectors

$$
\varepsilon_{i, h}^{\mu}=\frac{1}{\sqrt{2}}\left(\varepsilon_{i, X}^{\mu}+h i \varepsilon_{i, Y}^{\mu}\right),
$$


polarized along two directions $X$ and $Y$. The polarization vector along $X$ is defined through the following ansatz

$$
\varepsilon_{i, X}^{\mu}=c_{i, 1}^{X} p_{1}^{\mu}+c_{i, 2}^{X} p_{2}^{\mu}+c_{i, 3}^{X} p_{i}^{\mu} .
$$

As a reference vector for all vectors $\varepsilon_{i, X}^{\mu}$ we chose the vector $q^{\mu}=p_{1}^{\mu}+p_{2}^{\mu}$. The coefficients $c_{i, n}^{X}, n=1,2,3$ are determined from the system of normalization and orthogonality conditions for the polarization vectors:

$$
\left(\varepsilon_{i, X}\right)^{2}=-1, \quad \varepsilon_{i, X} \cdot q=0, \quad \varepsilon_{i, X} \cdot p_{i}=0 .
$$

The polarization vector along the direction $Y$ is given by

$$
\varepsilon_{i, Y}^{\mu}=\mathcal{N}_{i, Y} \varepsilon_{\nu \rho \sigma}^{\mu} q^{\nu} p_{i}^{\rho} \varepsilon_{i, X}^{\sigma}
$$

where we have used the conventions of ref. [79] for the Levi-Civita symbol: $\varepsilon^{0123}=+1$ and $\varepsilon_{0123}=-\varepsilon^{0123}$. The normalization factors $\mathcal{N}_{i, Y}$ are determined from the condition $\varepsilon_{i, Y}^{2}=-1$.

Lastly, we note that the above construction does not fix the vectors $\varepsilon_{i, X}$ and $\varepsilon_{i, Y}$ uniquely. There are two possible solutions which correspond to the change $\varepsilon_{i, X / Y} \rightarrow-\varepsilon_{i, X / Y}$. The overall signs of these two vectors are chosen in such a way that the vectors $\vec{p}_{i}, \vec{\varepsilon}_{i, X}$ and $\vec{\varepsilon}_{i, Y}$ form a right-handed coordinate system.

The fermion wave functions are treated in the following way. The spinor part of the amplitude (2.23) has the following structure

$$
\mathcal{M}=\bar{v}\left(p_{2}, h_{2}\right) \Gamma u\left(p_{1}, h_{1}\right)=\operatorname{Tr}\{(u \otimes \bar{v}) \Gamma\}
$$

The matrix $u \otimes \bar{v}$ in the above equation can be rewritten in the following way:

$$
(u \otimes \bar{v})_{\alpha \beta}=\frac{\bar{u} N v}{\bar{u} N v}(u \otimes \bar{v})_{\alpha \beta}=\frac{1}{\bar{u} N v}(u \otimes \bar{u})_{\alpha \gamma} N_{\gamma \delta}(v \otimes \bar{v})_{\delta \beta}=\frac{1}{\mathcal{N}}[(u \otimes \bar{u}) N(v \otimes \bar{v})]_{\alpha \beta},
$$

for some matrix $N$, to be specified below, and $\mathcal{N} \equiv \bar{u} N v \neq 0$. The outer spinor products read

$$
\begin{aligned}
& u\left(p, h_{1}\right) \otimes \bar{u}\left(p, h_{1}\right)=\not p \frac{1-h_{1} \gamma_{5}}{2}, \\
& v\left(p, h_{2}\right) \otimes \bar{v}\left(p, h_{2}\right)=\frac{1-h_{2} \gamma_{5}}{2} \not p .
\end{aligned}
$$

The matrix $N$ depends on the process-specific kinematics. In particular, it is linearly independent of $p_{1}$ and $p_{2}$, otherwise $\bar{u} N v=0$. Since for helicity configurations with $h_{1}=h_{2}$ the amplitude (2.1) vanishes to all orders, in the following we only consider the case $h=h_{1}=-h_{2}$. For this helicity configuration the matrix $N$ is given by

$$
N=i \varepsilon_{\gamma p_{3} p_{4} p_{5}} \text { if } \quad h_{1} \neq h_{2} .
$$


For this choice of $N$, eq. (2.31) takes the form

$$
\begin{aligned}
u \otimes \bar{v} & =\frac{1}{\mathcal{N}} \not p_{1} \frac{1-h \gamma_{5}}{2} i \varepsilon_{\gamma p_{3} p_{4} p_{5}} \frac{1+h \gamma_{5}}{2} \not p_{2} \\
& =\frac{1}{\mathcal{N}}\left(\frac{1}{2} \not p_{1} i \varepsilon_{\gamma p_{3} p_{4} p_{5} \not p_{2}}-\frac{h}{2} \not p_{1}\left(\frac{1}{3 !} \varepsilon_{\gamma \gamma \gamma \mu} \varepsilon^{\mu p_{3} p_{4} p_{5}}\right) \not p_{2}\right) \\
& =\frac{1}{\mathcal{N}}\left(\frac{1}{2} \not p_{1} i \varepsilon_{\gamma p_{3} p_{4} p_{5}} \not p_{2}+\frac{h}{2} \not p_{1} \frac{1}{3 !}\left(\sum_{i \neq j \neq k=3,4,5}(-1)^{N_{\mathrm{perm}}[i, j, k]} \not p_{i} \not p_{j} \not p_{k}\right) \not p_{2}\right)
\end{aligned}
$$

where $N_{\text {perm }}[i, j, k]$ is the number of transpositions needed to map an ordering $(i, j, k)$ to the default ordering $(i, j, k)=(3,4,5)$.

As mentioned, in the above equation we have used the relations $\left\{\gamma^{\mu}, \gamma_{5}\right\}=0$ and $\gamma_{5} \gamma^{\sigma}=\frac{-i}{3 !} \varepsilon^{\mu \nu \rho \sigma} \gamma_{\mu} \gamma_{\nu} \gamma_{\rho}$, and all manipulations in eq. (2.35) have been done in 4 dimensions. The normalization factor $\mathcal{N}$ needs to be evaluated only after IR renormalization, in the context of the finite remainder $\mathcal{F}$. For this reason the calculation of $\mathcal{N}$ is straightforward and can also be performed in 4 dimensions.

To summarize the results of this section, the amplitude for a specific helicity configuration eq. (2.23) is obtained by evaluating the photon polarization vectors according to eq. (2.26) and the product of the two quark spinors according to eq. (2.35). The resulting expressions are contracted with the tensor $\Gamma_{\mu \nu \rho}$ which is constructed diagrammatically and whose expression is independent of helicities. In practice only the two helicity configurations shown in eq. (2.22) need to be computed. The generation of the Feynman diagrams is performed with the help of a private software and the calculations of spinor traces and color factors are performed with the help of the program FORM [84].

\subsection{Reduction to pentagon functions}

In this work we consider four independent amplitude structures. They correspond to the two color factors in eq. (2.21) and the two helicities in eq. (2.22). We would like to express these structures in terms of transcendental functions and transcendental constants with rational coefficients. Since we assume that the set of functions forms a basis, the evaluation of an amplitude structure is equivalent to the evaluation of all its rational coefficients. To this end, we have built an automated framework that uses finite-field methods to numerically evaluate, interpolate and then reconstruct the exact analytical expressions for these coefficients. Throughout this section, the term numerical should be understood to refer to finite-field numerics. We will now provide a detailed account of our framework.

Any bare scalar 2-loop amplitude, or amplitude structure, $\mathcal{M}$ is expressed as a linear combination of 2-loop scalar integrals $I_{b}$ :

$$
\mathcal{M}=\sum_{b} A_{b} I_{b}
$$

The coefficients $A_{b}$ are rational functions of the kinematic invariants $s_{i j}$, defined in section 2.1, and polynomials in $\varepsilon$. As explained in section 2.2 the coefficients $A_{b}$ are also 


\begin{tabular}{|cc|}
\hline$i$ & $\Pi_{i}$ \\
\hline 1 & $k_{1}^{2}$ \\
2 & $k_{2}^{2}$ \\
3 & $\left(k_{1}+p_{1}+p_{2}\right)^{2}$ \\
4 & $\left(k_{1}-k_{2}\right)^{2}$ \\
5 & $\left(k_{2}+p_{1}\right)^{2}$ \\
6 & $\left(k_{2}+p_{1}+p_{2}\right)^{2}$ \\
7 & $\left(k_{2}-p_{3}\right)^{2}$ \\
8 & $\left(k_{1}+p_{1}+p_{2}-p_{3}\right)^{2}$ \\
9 & $\left(k_{1}+p_{1}+p_{2}-p_{3}-p_{4}\right)^{2}$ \\
10 & $\left(k_{2}-p_{3}-p_{4}\right)^{2}$ \\
11 & $\left(k_{1}+p_{1}\right)^{2}$ \\
\hline
\end{tabular}

Table 1. The propagators $\left\{\Pi_{i}\right\}$ defining the 2-loop 5-point planar integrals $I_{b}$.

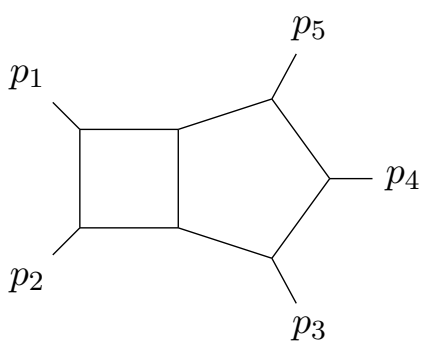

$C_{1}$

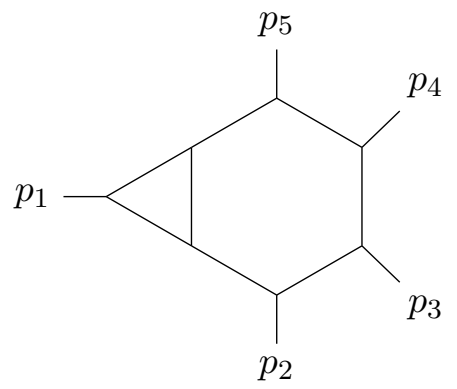

$\mathrm{C}_{2}$

Figure 2. The integral topologies $C_{1}$ and $C_{2}$.

linear functions of the parity-odd kinematic variable $\operatorname{tr}_{5}$. The planar two-loop integrals $I_{b}$ appearing in the amplitude of interest in this paper are defined [43] as follows:

$$
I_{b}=\int \frac{d^{d} k_{1} d^{d} k_{2}}{\Pi_{1}^{n_{1}} \Pi_{2}^{n_{2}} \ldots \Pi_{11}^{n_{11}}}
$$

where the propagators $\Pi_{i}$ are defined in table 1 and $n_{i} \equiv n_{i}(b) \in \mathbb{Z}$. These 11 propagators form a complete basis of bilinears through which any scalar numerator structure in an integrand can be expressed. A maximum of 8 propagators appear in the denominator of any given integrand; for the three remaining propagators, denoted spurious propagators, the corresponding indices $n_{i}$ satisfy $n_{i} \leq 0$. We can accordingly classify the integrals into two topologies, which are represented pictorially in figure 2 . In the $C_{1}$ topology, propagators 7,10 , and 11 are spurious. In the $C_{2}$ topology, propagators 6,7 , and 10 are spurious.

Following the standard Integration-By-Parts (IBP) approach, we map the integrals $I_{b}$ onto a basis of master integrals, $M_{c}$

$$
I_{b}=\sum_{c} B_{b c} M_{c}
$$


The coefficients $B_{b c}$ are rational functions of $s_{i j}$ and $\varepsilon$. All coefficients required in this calculation are known analytically from ref. [43]. The IBP identities map all required $C_{1}$ integrals onto a basis of 61 master integrals, ${ }^{1}$ and all required $C_{2}$ integrals onto a basis of 28 master integrals. The $28 C_{2}$ master integrals can themselves be identified with $C_{1}$ integrals, in some cases by permuting the external momenta $\left\{p_{j}\right\}$.

The next step in our calculation is to map the master integrals $M_{c}$ onto a basis of transcendental functions and constants. In this work we choose the basis of functions provided recently in ref. [59]. That reference solves a set of specially designed loop master integrals $M^{(\mathrm{UT})}$ of uniform transcendentality (UT)

$$
M_{d}^{(\mathrm{UT})}=\sum_{e} D_{d e} t_{e}
$$

where the basis $\left\{t_{e}\right\}$ is built out of sums and products of the transcendental functions $\mathrm{F}$ and transcendental constants tcr and tci that are defined in ref. [59] as well as the unity. The coefficients $D_{d e}$ are rational functions of the kinematic invariants $s_{i j}$ and linear functions of $\operatorname{tr}_{5}$. They also depend on $\varepsilon$, however, they are only known as a series expansion of sufficient depth

$$
D_{d e}=\sum_{n=-4}^{0} D_{d e}^{(n)} \varepsilon^{n}+\mathcal{O}(\varepsilon) .
$$

In order to map our set of master integrals $M_{c}$ onto the function basis $\left\{t_{e}\right\}$ we first use the analytic form of the IBP solutions from ref. [43] to express the set $M^{(\mathrm{UT})}$ in terms of the set $M_{c}$, then we invert it:

$$
M_{c}=\sum_{d} B_{c d}^{(\mathrm{UT})} M_{d}^{(\mathrm{UT})}
$$

Combining equations (2.41) and (2.39), we obtain an analytical expression for each $C_{1}$ master integral $M_{c}$ in terms of the function basis $\left\{t_{e}\right\}$

$$
M_{c}=\sum_{e} E_{c e} t_{e}
$$

where

$$
E_{c e}=\sum_{d} B_{c d}^{(\mathrm{UT})} D_{d e}
$$

We would like to combine the above results in order to express the amplitude $\mathcal{M}$ in terms of the functions $t_{e}$ :

$$
\mathcal{M}=\sum_{e} G_{e} t_{e}
$$

with coefficients

$$
G_{e}=\sum_{b} \sum_{c} A_{b} B_{b c} E_{c e}
$$

\footnotetext{
${ }^{1}$ For the purposes of the IBP-solving approach in ref. [43] there are 62 master integrals in the $C_{1}$ topology, but two of these are related to each other by a discrete symmetry and can be set equal in the present context.
} 
In practice, we only seek the Laurent expansion $G_{e}^{(n)}$ of the coefficients $G_{e}$

$$
G_{e}=\sum_{n=-4}^{0} G_{e}^{(n)} \varepsilon^{n}+\mathcal{O}(\varepsilon),
$$

where

$$
G_{e}^{(n)}=\sum_{n_{1}+n_{2}+n_{3}=n} \sum_{b} \sum_{c} A_{b}^{\left(n_{1}\right)} B_{b c}^{\left(n_{2}\right)} E_{c e}^{\left(n_{3}\right)} .
$$

The coefficient $A_{b}^{(n)}$ appearing in eq. (2.47) are defined as

$$
A_{b}=\sum_{n=n_{\min }}^{n_{\max }} A_{b}^{(n)} \varepsilon^{n}
$$

The coefficients $B_{b c}^{(n)}$ and $E_{c e}^{(n)}$ appearing in eq. (2.47) are defined similarly to eq. (2.48) but with respect to the functions $B_{b c}$ and $E_{c e}$. For each one of the functions $A_{b}, B_{b c}$ and $E_{c e}$, the powers $n_{\min }$ and $n_{\max }$ appearing in eq. (2.48) are chosen in such a way that the range for the index $n$ in eq. (2.46) is satisfied.

The coefficients $G_{e}^{(n)}$ can be split into two parts: one which is proportional to $\operatorname{tr}_{5}$ and one which is independent of it. Both parts are rational functions of the invariants $s_{i j}$. The reason only the first power of the parity-odd variable $\operatorname{tr}_{5}$ appears in the final result is that $\left(\operatorname{tr}_{5}\right)^{2}$ is itself a polynomial of $s_{i j}$, see eq. (2.9).

The calculation of the coefficients $G_{e}^{(n)}$ is based on eq. (2.47) and proceeds as follows. We first note that the coefficients $A_{b}^{\left(n_{1}\right)}, B_{b c}^{\left(n_{2}\right)}$ and $E_{c e}^{\left(n_{3}\right)}$ appearing in that equation are all known in analytic form. In principle one can multiply and add them, as required, to derive the analytic expressions of the coefficients $G_{e}^{(n)}$. The problem with this strategy is that the size of the rational expressions that need to be combined becomes huge which hampers their subsequent simplification. Such a strategy was followed by us in ref. [33] for the evaluation of the squared amplitude for $q \bar{q} \rightarrow \gamma \gamma \gamma$. We refer the reader to that reference for more details about the subtleties of such an approach.

In this work we follow an alternative strategy for the evaluation of the coefficients $G_{e}^{(n)}$. The idea is to numerically evaluate the functions $A_{b}^{\left(n_{1}\right)}, B_{b c}^{\left(n_{2}\right)}$ and $E_{c e}^{\left(n_{3}\right)}$, then multiply and add them as appropriate, in order to obtain a numerical value for $G_{e}^{(n)}$ in a given kinematic point. The finite-field evaluations of $G_{e}^{(n)}$ are then passed to the FireFly library [85] in order to reconstruct the exact analytical expressions for the coefficients $G_{e}^{(n)}$.

We have created an automated framework which is designed to calculate simultaneously multiple amplitudes (that have the same kinematics) following the finite-field evaluation approach just described. The reason it may be advantageous to compute several amplitudes at the same time is efficiency, noting that only the coefficients $A_{b}^{\left(n_{1}\right)}$ depend on the amplitude while the coefficients $B_{b c}^{\left(n_{2}\right)}$ and $E_{c e}^{\left(n_{3}\right)}$ are process independent.

To fully specify our approach we need to describe one more feature which has to do with momentum crossings. The coefficients $A_{b}^{\left(n_{1}\right)}$ do not require any further crossing since all crossings are already included at the diagrammatic level. Similarly, the functions $\left\{t_{e}\right\}$ are already defined in such a way that all possible crossings have been already implemented 
in their definition [59]. This is one significant difference with respect to the functional basis constructed in ref. [47] and used by us in ref. [33]. The basis of ref. [47] does not include momentum crossings and if they are required the user needs to implement those. As a result of such crossings one generally arrives at a functional basis which is non-minimal. To reduce the extended set of functions to a minimal set, functional identities need to be derived and applied; see ref. [33] for more details on this point.

Where momentum crossings still need to be applied is the part of eq. (2.47) that involves IBPs. Specifically, this affects the coefficients $B_{b c}^{\left(n_{2}\right)}$ as well as the coefficients $E_{c e}^{\left(n_{3}\right)}$ through their constituent coefficients $B_{c d}^{(\mathrm{UT})}$, see eq. (2.41). The reason additional crossings are needed in the parts where IBPs are involved is that, in general, an integral can appear in an amplitude with any of the 5! permutations of the external legs while the analytical IBP solutions are only needed - and therefore only provided - for the 'standard', topology-defining permutation eq. (2.37), see also table 1 and figure 2. In principle any crossing of the IBP solution can be obtained analytically by rearranging the solution in terms of the crossed kinematic invariants. Such a strategy would be impractical in our numerical approach due to the significant size of the IBP solutions and the very large number of crossings.

The way we deal with crossings in our practical implementation is as follows. The master integral reductions $E_{c e}^{(n)}$ are derived analytically for all required momentum crossings. The coefficients $B_{b c}^{\left(n_{2}\right)}$ are permuted numerically by first applying the corresponding permutation to the numerical values of the kinematic invariants $s_{i j}$ and then evaluating the 'standard' IBP solutions at the resulting numerical point. Since the coefficients $B_{b c}^{\left(n_{2}\right)}$ are universal, at each finite-field point they only need to be evaluated once per required momentum crossing, even if an integral $I_{b}$ appears in several amplitudes. Due to the large number of integrals and crossings, it is impractical to store the full set of numerical values $B_{b c}^{\left(n_{2}\right)}$, even for just a single finite-field point per computing thread. ${ }^{2}$ Instead, after evaluating the coefficients $B_{b c}^{\left(n_{2}\right)}$, we immediately multiply by $A_{b}^{\left(n_{1}\right)}$ for all amplitudes $\mathcal{M}$ and we only store running totals of $\sum_{b} A_{b}^{\left(n_{1}\right)} B_{b c}^{\left(n_{2}\right)}$.

\section{Results}

Following the approach described in the previous section, in this work we calculate in analytical form the two helicities eq. (2.22) of the finite remainder for the process $q \bar{q} \rightarrow \gamma \gamma \gamma$ in the 't Hooft-Veltman scheme. In this work we have not included any non-planar contributions i.e. we work in the approximation eq. (2.20). Factoring out the phase-dependent part of the leading order amplitude we write the reconstructed finite remainder for the helicity $\bar{h}_{+}$defined in eq. $(2.22)$ as:

$$
\mathcal{F}^{\bar{h}_{+}}=\mathcal{F}^{\bar{h}_{+}(0)}\left(1+\frac{\alpha_{s}}{4 \pi} C_{F} \mathcal{R}^{\bar{h}_{+}(1)}+\left(\frac{\alpha_{s}}{4 \pi}\right)^{2}\left(N_{c}^{2} \mathcal{R}^{\bar{h}_{+}\left(N_{c}^{2}\right)}+C_{F} n_{f} \mathcal{R}^{\bar{h}_{+}\left(C_{F} n_{f}\right)}\right)+\mathcal{O}\left(\alpha_{s}^{3}\right)\right)
$$

\footnotetext{
${ }^{2}$ We use a multi-core computing cluster. Each computing thread is assigned independent finite-field points and evaluates all the amplitudes at the assigned points, one point at time.
} 
For the helicity $\bar{h}_{-}$the tree-level amplitude vanishes and we write

$$
\mathcal{F}^{\bar{h}_{-}}=\tilde{\mathcal{F}}^{\bar{h}_{-}}\left(\frac{\alpha_{s}}{4 \pi} C_{F} \mathcal{R}^{\bar{h}_{-}(1)}+\left(\frac{\alpha_{s}}{4 \pi}\right)^{2}\left(N_{c}^{2} \mathcal{R}^{\bar{h}_{-}\left(N_{c}^{2}\right)}+C_{F} n_{f} \mathcal{R}^{\bar{h}_{-}\left(C_{F} n_{f}\right)}\right)+\mathcal{O}\left(\alpha_{s}^{3}\right)\right) .
$$

The functions $\mathcal{R}^{\bar{h}(i)}, i=\left(1, N_{c}^{2}, C_{F} n_{f}\right)$, have the following structure

$$
\mathcal{R}^{\bar{h}(i)}=\sum_{e} r_{e}^{\bar{h}(i)} t_{e}
$$

They are independent of the specific phase choices made in the construction of the external wave-functions. The coefficients $r_{e}^{\bar{h}(i)}$ appearing in eq. (3.3) are rational functions of the parity-even invariants $s_{i j}$ and are linear functions of $\operatorname{tr}_{5}$. The explicit expressions for the functions $\mathcal{R}^{\bar{h}(i)}$, as well as for the tree-level amplitude $\mathcal{F}^{\bar{h}_{+}(0)}$ and for the phase-dependent factor $\tilde{\mathcal{F}}^{\bar{h}_{-}}$can be found in electronic form in the ancillary files accompanying the arXiv submission of this article.

We have checked that the above result agrees numerically with our previous calculation performed in ref. [33]. This comparison does not include the terms $\sim n_{f}$ since those were not computed in ref. [33]. Given the two calculations were performed with almost completely independent methods this represents a strong check on the correctness of eqs. (3.1), (3.2). Since one of the main objectives of the present work is to document the calculation of the two-loop amplitude used in ref. [33], we will next explain in some detail how the two calculational approaches differ from each other.

Ref. [33] computed directly the squared amplitude, while in this work we compute the helicity amplitudes and the squared amplitude is obtained by squaring and crossing them numerically. Ref. [33] used the polygon functional basis of ref. [47] while in the present work we use the basis of ref. [59]. The main difference between the two functional bases was explained in the previous section. Our calculation in ref. [33] was fully analytic while the current calculation uses finite-field numeric evaluation and reconstruction techniques to obtain the rational coefficients. Besides the differences already mentioned, the two results differ significantly as far as evaluation times are concerned. The main reason for this is the difference in evaluation times and numerical precision between the two functional bases. The larger size of the result in ref. [33] is immaterial given the time needed for the evaluation of the most complicated functions in that basis.

We have also checked that our results eqs. (3.1), (3.2) agree with the results in ref. [78]. Since we have computed a different helicity combination relative to the helicities published in ref. [78], a direct analytic comparison between the two is complicated by the fact crossing of external legs is required. We have analytically checked most structures which are simple enough to cross, while the complete expressions (for each helicity and color factor) have been compared numerically with high numerical precision (30 digits) and full agreement between the two calculations has been found in all cases. There are certain differences in the way the present calculation and the one in ref. [78] were performed that make the agreement between the two calculations highly nontrivial. First, the generation of diagrams is based on different approaches. Furthermore, in this work we use the analytic solutions of the IBP equations from ref. [43] while ref. [78] solves the IBP equations numerically for 
each point in which the amplitude is evaluated and being reconstructed. Most importantly, in this work we use an alternative approach to the projection of helicity amplitudes which is very different from the one employed in ref. [78].

\section{Conclusion}

In this work we calculate the planar contributions to the two-loop helicity amplitude for the process $q \bar{q} \rightarrow \gamma \gamma \gamma$. The result is presented in fully analytic form and is available for download in electronic form with the arXiv submission of this paper. This result, written in an alternative form, was used in the first NNLO calculation of a $2 \rightarrow 3$ LHC process [33].

The helicity amplitudes are expressed in terms of the functional basis of ref. [59] which allows fast and efficient numerical evaluation of the amplitudes. These functions' speed of evaluation is sufficiently high to allow the direct use of this amplitude in the calculation of NNLO cross-sections without the need for intermediate interpolation.

In our calculation we have utilized Chen's recently proposed approach [79] for the efficient projection of helicity amplitudes in multileg/multiloop processes. We have found the approach very easy to implement and use especially since it does not involve investigations of tensor bases that grow with the number of loops.

We have found complete agreement between our calculation and the recent independent calculation of the same amplitude in ref. [78]. Such an agreement represents a highly nontrivial check on both calculations given they use very different computational approaches.

Our calculation is derived from an automated framework we have created for the calculation of generic two-loop massless 5-point gauge theory amplitudes. We hope it will prove suitable for many other potential applications.

\section{Acknowledgments}

We acknowledge helpful discussions with Long Chen about the helicity projection method of ref. [79] and with Fabian Lange and Jonas Klappert on the use of the library FireFly [85]. The work of M.C. was supported by the Deutsche Forschungsgemeinschaft under grant 396021762 - TRR 257. The research of H.C., A.M. and R.P. has received funding from the European Research Council (ERC) under the European Union's Horizon 2020 Research and Innovation Programme (grant agreement no. 683211). A.M. was also supported by the U.K. STFC grants ST/L002760/1 and ST/K004883/1. The research of H.C. has also received funding under the ERC grant agreement 804394. A.M. acknowledges the use of the DiRAC Cumulus HPC facility under Grant No. PPSP226.

\section{A Renormalization constants}

The UV renormalisation constant of the quark wave function through order $\mathcal{O}\left(\alpha_{s}^{2}\right)$ reads

$$
Z_{q}=1+\left(\frac{\alpha_{s}}{4 \pi}\right)^{2}\left(C_{F} T_{F}\left(-\frac{5}{6}+\frac{1}{\varepsilon}\right)\right)
$$


while the renormalization constant $Z_{\alpha_{s}}=Z_{g}^{2}$ up to power $\alpha_{s}$ (higher powers are not required since the tree-level amplitude for $p p \rightarrow \gamma \gamma \gamma$ has a zero power of $\alpha_{s}$ ) is given by

$$
Z_{g}=1+\frac{1}{\varepsilon}\left(\frac{\alpha_{s}}{4 \pi}\right) \frac{4\left(n_{l}+1\right) T_{F}-11 C_{A}}{6} .
$$

The contribution from heavy flavours is not considered in this work, therefore we have $Z_{q}=1$ and the term $\left(n_{l}+1\right)$ term in $Z_{g}$ reads $n_{l}$.

For the IR renormalization of the amplitude for the process $q \bar{q} \rightarrow \gamma \gamma \gamma$ the color-space matrix $\mathbf{Z}$ is needed. Through order $\mathcal{O}\left(\alpha_{s}^{2}\right)$ it is given by

$$
\begin{aligned}
\mathbf{Z}= & +\left(\frac{\alpha_{s}}{4 \pi}\right)\left(-\frac{2 C_{F}}{\varepsilon^{2}}-\frac{2 C_{F} l_{\mu}+3 C_{F}}{\varepsilon}\right) \\
& +\left(\frac{\alpha_{s}}{4 \pi}\right)^{2}\left(\frac{2 C_{F}^{2}}{\varepsilon^{4}}+\frac{C_{F}\left(11 C_{A}+8 C_{F} l_{\mu}+12 C_{F}-4 n_{l} T_{F}\right)}{2 \varepsilon^{3}}\right. \\
& +\frac{C_{F}\left(6 l_{\mu}\left(11 C_{A}+18 C_{F}-4 n_{l} T_{F}\right)+2 C_{A}\left(9 \zeta_{2}+16\right)+36 C_{F} l_{\mu}^{2}+81 C_{F}-16 n_{l} T_{F}\right)}{18 \varepsilon^{2}} \\
& +\frac{C_{F}\left(12 l_{\mu}\left(C_{A}\left(18 \zeta_{2}-67\right)+20 n_{l} T_{F}\right)+C_{A}\left(1404 \zeta_{3}-594 \zeta_{2}-961\right)-1296 C_{F} \zeta_{3}\right.}{108 \varepsilon} \\
& \left.+\frac{\left.648 C_{F} \zeta_{2}-81 C_{F}+216 n_{l} T_{F} \zeta_{2}+260 n_{l} T_{F}\right)}{108 \varepsilon}\right),
\end{aligned}
$$

with the abbreviation $l_{\mu}=\log \left(-\mu^{2} / s_{12}\right)$.

Open Access. This article is distributed under the terms of the Creative Commons Attribution License (CC-BY 4.0), which permits any use, distribution and reproduction in any medium, provided the original author(s) and source are credited.

\section{References}

[1] D. Chicherin, J.M. Henn and E. Sokatchev, Amplitudes from anomalous superconformal symmetry, JHEP 01 (2019) 179 [arXiv:1811.02560] [INSPIRE].

[2] G. Kälin, G. Mogull and A. Ochirov, Two-loop $\mathcal{N}=2$ SQCD amplitudes with external matter from iterated cuts, JHEP 07 (2019) 120 [arXiv:1811.09604] [INSPIRE].

[3] S. Abreu, L.J. Dixon, E. Herrmann, B. Page and M. Zeng, The two-loop five-point amplitude in $\mathcal{N}=4$ super-Yang-Mills theory, Phys. Rev. Lett. 122 (2019) 121603 [arXiv:1812.08941] [INSPIRE].

[4] D. Chicherin, T. Gehrmann, J.M. Henn, P. Wasser, Y. Zhang and S. Zoia, Analytic result for a two-loop five-particle amplitude, Phys. Rev. Lett. 122 (2019) 121602 [arXiv:1812.11057] [INSPIRE].

[5] S. Abreu, L.J. Dixon, E. Herrmann, B. Page and M. Zeng, The two-loop five-point amplitude in $\mathcal{N}=8$ supergravity, JHEP 03 (2019) 123 [arXiv:1901.08563] [INSPIRE]. 
[6] A. Klemm, C. Nega and R. Safari, The l-loop Banana Amplitude from GKZ Systems and relative Calabi-Yau Periods, JHEP 04 (2020) 088 [arXiv: 1912.06201] [INSPIRE].

[7] J.L. Bourjaily, E. Herrmann, C. Langer, A.J. McLeod and J. Trnka, All-Multiplicity Nonplanar Amplitude Integrands in Maximally Supersymmetric Yang-Mills Theory at Two Loops, Phys. Rev. Lett. 124 (2020) 111603 [arXiv:1911.09106] [INSPIRE].

[8] B. Basso, L.J. Dixon and G. Papathanasiou, Origin of the Six-Gluon Amplitude in Planar $N=4$ Supersymmetric Yang-Mills Theory, Phys. Rev. Lett. 124 (2020) 161603 [arXiv: 2001.05460] [INSPIRE].

[9] C. Duhr, H. Johansson, G. Kälin, G. Mogull and B. Verbeek, Full-Color Two-Loop Four-Gluon Amplitude in $\mathcal{N}=2$ Supersymmetric QCD, Phys. Rev. Lett. 123 (2019) 241601 [arXiv: 1904.05299] [INSPIRE].

[10] J. Bartels, Analytic structure of the 8-point scattering amplitude in multi-Regge kinematics in $N=4$ SYM: conformal Regge pole and Regge cut contributions, arXiv:2005.08818 [INSPIRE].

[11] N. Arkani-Hamed, T. Lam and M. Spradlin, Non-perturbative geometries for planar $\mathcal{N}=4$ SYM amplitudes, JHEP 03 (2021) 065 [arXiv: 1912.08222] [INSPIRE].

[12] S. Caron-Huot et al., The Steinmann Cluster Bootstrap for $N=4$ Super Yang-Mills Amplitudes, PoS CORFU2019 (2020) 003 [arXiv:2005.06735] [INSPIRE].

[13] J.M. Henn, G.P. Korchemsky and B. Mistlberger, The full four-loop cusp anomalous dimension in $\mathcal{N}=4$ super Yang-Mills and QCD, JHEP 04 (2020) 018 [arXiv:1911.10174] [INSPIRE].

[14] S. Abreu et al., Two-Loop Four-Graviton Scattering Amplitudes, Phys. Rev. Lett. 124 (2020) 211601 [arXiv: 2002 .12374] [INSPIRE].

[15] S. Banerjee, S. Ghosh, P. Pandey and A.P. Saha, Modified celestial amplitude in Einstein gravity, JHEP 03 (2020) 125 [arXiv: 1909.03075] [INSPIRE].

[16] D. Chicherin, T. Gehrmann, J.M. Henn, P. Wasser, Y. Zhang and S. Zoia, The two-loop five-particle amplitude in $\mathcal{N}=8$ supergravity, JHEP 03 (2019) 115 [arXiv:1901.05932] [INSPIRE].

[17] A.R. Dalgleish, D.C. Dunbar, W.B. Perkins and J.M.W. Strong, Full color two-loop six-gluon all-plus helicity amplitude, Phys. Rev. D 101 (2020) 076024 [arXiv:2003.00897] [INSPIRE].

[18] D.C. Dunbar, J.H. Godwin, W.B. Perkins and J.M.W. Strong, Color Dressed Unitarity and Recursion for Yang-Mills Two-Loop All-Plus Amplitudes, Phys. Rev. D 101 (2020) 016009 [arXiv: 1911.06547] [INSPIRE].

[19] C. Anastasiou, R. Haindl, G. Sterman, Z. Yang and M. Zeng, Locally finite two-loop amplitudes for off-shell multi-photon production in electron-positron annihilation, JHEP 04 (2021) 222 [arXiv : 2008.12293] [INSPIRE].

[20] A.J. Larkoski, I. Moult and B. Nachman, Jet Substructure at the Large Hadron Collider: A Review of Recent Advances in Theory and Machine Learning, Phys. Rept. 841 (2020) 1 [arXiv: 1709.04464] [INSPIRE].

[21] S. Badger et al., Applications of integrand reduction to two-loop five-point scattering amplitudes in QCD, PoS LL2018 (2018) 006 [arXiv: 1807.09709] [INSPIRE]. 
[22] M.A. Lim, Quantum Chromodynamics and the Precision Phenomenology of Heavy Quarks, Ph.D. thesis, Cambridge U., 2018. arXiv:1811.10874. 10.17863/CAM.32479 [INSPIRE].

[23] S. Abreu, J. Dormans, F. Febres Cordero, H. Ita and B. Page, Analytic Form of Planar Two-Loop Five-Gluon Scattering Amplitudes in QCD, Phys. Rev. Lett. 122 (2019) 082002 [arXiv: 1812.04586] [INSPIRE].

[24] S. Abreu, J. Dormans, F. Febres Cordero, H. Ita, B. Page and V. Sotnikov, Analytic Form of the Planar Two-Loop Five-Parton Scattering Amplitudes in QCD, JHEP 05 (2019) 084 [arXiv: 1904.00945] [INSPIRE].

[25] H.B. Hartanto, S. Badger, C. Brønnum-Hansen and T. Peraro, A numerical evaluation of planar two-loop helicity amplitudes for a W-boson plus four partons, JHEP 09 (2019) 119 [arXiv: 1906.11862] [INSPIRE].

[26] A. Ochirov and B. Page, Multi-Quark Colour Decompositions from Unitarity, JHEP 10 (2019) 058 [arXiv : 1908.02695] [INSPIRE].

[27] D.C. Dunbar, W.B. Perkins and J.M.W. Strong, n-point QCD two-loop amplitude, Phys. Rev. D 101 (2020) 076001 [arXiv:2001.11347] [InSPIRE].

[28] R. Poncelet, NNLO QCD Calculations with the Sector-improved Residue Subtraction Scheme, Acta Phys. Polon. B 51 (2020) 1503 [inSPIRE].

[29] M. Czakon, A. Mitov and R. Poncelet, NNLO QCD corrections to leptonic observables in top-quark pair production and decay, JHEP 05 (2021) 212 [arXiv:2008.11133] [INSPIRE].

[30] T. Ahmed, A.H. Ajjath, G. Das, P. Mukherjee, V. Ravindran and S. Tiwari, Soft-virtual correction and threshold resummation for $n$-colorless particles to fourth order in QCD: Part I, arXiv:2010.02979 [INSPIRE].

[31] L. Magnea, G. Pelliccioli, C. Signorile-Signorile, P. Torrielli and S. Uccirati, Analytic integration of soft and collinear radiation in factorised QCD cross sections at NNLO, JHEP 02 (2021) 037 [arXiv: 2010.14493] [INSPIRE].

[32] A. Kardos, A.J. Larkoski and Z. Trócsányi, Two- and three-loop data for the groomed jet mass, Phys. Rev. D 101 (2020) 114034 [arXiv:2002.05730] [InSPIRE].

[33] H.A. Chawdhry, M.L. Czakon, A. Mitov and R. Poncelet, NNLO QCD corrections to three-photon production at the LHC, JHEP 02 (2020) 057 [arXiv: 1911.00479] [INSPIRE].

[34] L. Budge, J.M. Campbell, G. De Laurentis, R.K. Ellis and S. Seth, The one-loop amplitudes for Higgs +4 partons with full mass effects, JHEP 05 (2020) 079 [arXiv:2002.04018] [INSPIRE].

[35] P. Banerjee, S. Borowka, P.K. Dhani, T. Gehrmann and V. Ravindran, Two-loop massless $Q C D$ corrections to the $g+g \rightarrow H+H$ four-point amplitude, JHEP 11 (2018) 130 [arXiv: 1809. 05388] [INSPIRE].

[36] B. Agarwal and A. Von Manteuffel, On the two-loop amplitude for $g g \rightarrow Z Z$ production with full top-mass dependence, PoS RADCOR2019 (2019) 008 [arXiv:1912.08794] [inSPIRE].

[37] G. Bell, M. Beneke, T. Huber and X.-Q. Li, Two-loop non-leptonic penguin amplitude in QCD factorization, JHEP 04 (2020) 055 [arXiv:2002.03262] [INSPIRE].

[38] Y. Wang, Z. Li and N. Ul Basat, Direct reduction of multiloop multiscale scattering amplitudes, Phys. Rev. D 101 (2020) 076023 [arXiv: 1901.09390] [INSPIRE]. 
[39] T. Gehrmann, N. Glover, A. Huss and J. Whitehead, Scale and isolation sensitivity of diphoton distributions at the LHC, JHEP 01 (2021) 108 [arXiv:2009.11310] [INSPIRE].

[40] G. Heinrich, Collider Physics at the Precision Frontier, arXiv:2009.00516 [InSPIRE].

[41] ATLAS collaboration, Standard Model Summary Plots Spring 2020, ATL-PHYS-PUB-2020-010, CERN, Geneva (2020).

[42] S. Kallweit, V. Sotnikov and M. Wiesemann, Triphoton production at hadron colliders in NNLO QCD, Phys. Lett. B 812 (2021) 136013 [arXiv:2010.04681] [InSPIRE].

[43] H.A. Chawdhry, M.A. Lim and A. Mitov, Two-loop five-point massless QCD amplitudes within the integration-by-parts approach, Phys. Rev. D 99 (2019) 076011 [arXiv: 1805.09182] [INSPIRE].

[44] J. Böhm, A. Georgoudis, K.J. Larsen, H. Schönemann and Y. Zhang, Complete integration-by-parts reductions of the non-planar hexagon-box via module intersections, JHEP 09 (2018) 024 [arXiv: 1805.01873] [INSPIRE].

[45] A.V. Kotikov and S. Teber, Multi-loop techniques for massless Feynman diagram calculations, Phys. Part. Nucl. 50 (2019) 1 [arXiv: 1805.05109] [INSPIRE].

[46] J. Bosma, K.J. Larsen and Y. Zhang, Differential equations for loop integrals without squared propagators, PoS LL2018 (2018) 064 [arXiv:1807.01560] [INSPIRE].

[47] T. Gehrmann, J.M. Henn and N.A. Lo Presti, Pentagon functions for massless planar scattering amplitudes, JHEP 10 (2018) 103 [arXiv:1807.09812] [INSPIRE].

[48] S. Abreu, B. Page and M. Zeng, Differential equations from unitarity cuts: nonplanar hexa-box integrals, JHEP 01 (2019) 006 [arXiv:1807.11522] [INSPIRE].

[49] D. Chicherin, T. Gehrmann, J.M. Henn, N.A. Lo Presti, V. Mitev and P. Wasser, Analytic result for the nonplanar hexa-box integrals, JHEP 03 (2019) 042 [arXiv:1809.06240] [INSPIRE].

[50] P. Mastrolia and S. Mizera, Feynman Integrals and Intersection Theory, JHEP 02 (2019) 139 [arXiv: 1810.03818 ] [INSPIRE].

[51] P. Maierhöfer and J. Usovitsch, Kira 1.2 Release Notes, arXiv:1812.01491 [inSPIRE].

[52] A. Kardos, A new reduction strategy for special negative sectors of planar two-loop integrals without Laporta algorithm, arXiv: 1812.05622 [INSPIRE].

[53] H. Frellesvig et al., Decomposition of Feynman Integrals on the Maximal Cut by Intersection Numbers, JHEP 05 (2019) 153 [arXiv:1901.11510] [INSPIRE].

[54] D. Bendle et al., Integration-by-parts reductions of Feynman integrals using Singular and GPI-Space, JHEP 02 (2020) 079 [arXiv: 1908. 04301] [INSPIRE].

[55] C.G. Papadopoulos and C. Wever, Internal Reduction method for computing Feynman Integrals, JHEP 02 (2020) 112 [arXiv: 1910.06275] [INSPIRE].

[56] T. Peraro, Analytic multi-loop results using finite fields and dataflow graphs with FiniteFlow, in 14th International Symposium on Radiative Corrections: Application of Quantum Field Theory to Phenomenology, (2019), DOI [arXiv:1912.03142] [INSPIRE].

[57] X. Guan, X. Liu and Y.-Q. Ma, Complete reduction of integrals in two-loop five-light-parton scattering amplitudes, Chin. Phys. C 44 (2020) 093106 [arXiv:1912.09294] [INSPIRE]. 
[58] J. Usovitsch, Factorization of denominators in integration-by-parts reductions, arXiv: 2002.08173 [INSPIRE].

[59] D. Chicherin and V. Sotnikov, Pentagon Functions for Scattering of Five Massless Particles, JHEP 12 (2020) 167 [arXiv:2009.07803] [INSPIRE].

[60] D.D. Canko, C.G. Papadopoulos and N. Syrrakos, Analytic representation of all planar two-loop five-point Master Integrals with one off-shell leg, JHEP 01 (2021) 199 [arXiv:2009.13917] [INSPIRE].

[61] D. Bendle et al., Module Intersection for the Integration-by-Parts Reduction of Multi-Loop Feynman Integrals, in MathemAmplitudes 2019: Intersection Theory and Feynman Integrals, (2020) [arXiv: 2010.06895] [INSPIRE].

[62] S. Moch, J.A.M. Vermaseren and A. Vogt, Three-loop results for quark and gluon form-factors, Phys. Lett. B 625 (2005) 245 [hep-ph/0508055] [INSPIRE].

[63] P.A. Baikov, K.G. Chetyrkin, A.V. Smirnov, V.A. Smirnov and M. Steinhauser, Quark and gluon form factors to three loops, Phys. Rev. Lett. 102 (2009) 212002 [arXiv:0902.3519] [INSPIRE].

[64] T. Gehrmann, E.W.N. Glover, T. Huber, N. Ikizlerli and C. Studerus, Calculation of the quark and gluon form factors to three loops in QCD, JHEP 06 (2010) 094 [arXiv:1004.3653] [INSPIRE].

[65] J.M. Henn, A.V. Smirnov and V.A. Smirnov, Analytic results for planar three-loop four-point integrals from a Knizhnik-Zamolodchikov equation, JHEP 07 (2013) 128 [arXiv:1306.2799] [INSPIRE].

[66] T. Ahmed, J. Henn and B. Mistlberger, Four-particle scattering amplitudes in QCD at NNLO to higher orders in the dimensional regulator, JHEP 12 (2019) 177 [arXiv: 1910.06684] [INSPIRE].

[67] J. Henn, B. Mistlberger, V.A. Smirnov and P. Wasser, Constructing d-log integrands and computing master integrals for three-loop four-particle scattering, JHEP 04 (2020) 167 [arXiv: 2002.09492] [INSPIRE].

[68] F. Caola, A. Von Manteuffel and L. Tancredi, Diphoton Amplitudes in Three-Loop Quantum Chromodynamics, Phys. Rev. Lett. 126 (2021) 112004 [arXiv:2011.13946] [INSPIRE].

[69] S. Abreu, F. Febres Cordero, H. Ita, B. Page and M. Zeng, Five-Point Two-Loop Amplitudes from Numerical Unitarity, PoS LL2018 (2018) 016 [arXiv: 1807.09447] [INSPIRE].

[70] S. Abreu, F. Febres Cordero, H. Ita, B. Page and V. Sotnikov, Planar Two-Loop Five-Parton Amplitudes from Numerical Unitarity, JHEP 11 (2018) 116 [arXiv:1809.09067] [INSPIRE].

[71] S. Badger, C. Brønnum-Hansen, H.B. Hartanto and T. Peraro, Analytic helicity amplitudes for two-loop five-gluon scattering: the single-minus case, JHEP 01 (2019) 186 [arXiv: 1811.11699] [INSPIRE].

[72] S. Abreu et al., Caravel: A C++ Framework for the Computation of Multi-Loop Amplitudes with Numerical Unitarity, arXiv:2009.11957 [INSPIRE].

[73] G. De Laurentis and D. Maître, Two-Loop Five-Parton Leading-Colour Finite Remainders in the Spinor-Helicity Formalism, JHEP 02 (2021) 016 [arXiv: 2010.14525] [INSPIRE]. 
[74] D. Chicherin, T. Gehrmann, J.M. Henn, P. Wasser, Y. Zhang and S. Zoia, All Master Integrals for Three-Jet Production at Next-to-Next-to-Leading Order, Phys. Rev. Lett. 123 (2019) 041603 [arXiv: 1812.11160] [INSPIRE].

[75] S. Badger et al., Analytic form of the full two-loop five-gluon all-plus helicity amplitude, Phys. Rev. Lett. 123 (2019) 071601 [arXiv: 1905.03733] [INSPIRE].

[76] J. Boehm, M. Wittmann, Z. Wu, Y. Xu and Y. Zhang, IBP reduction coefficients made simple, JHEP 12 (2020) 054 [arXiv: 2008.13194] [INSPIRE].

[77] J. Klappert, F. Lange, P. Maierhöfer and J. Usovitsch, Integral reduction with Kira 2.0 and finite field methods, Comput. Phys. Commun. 266 (2021) 108024 [arXiv:2008.06494] [INSPIRE].

[78] S. Abreu, B. Page, E. Pascual and V. Sotnikov, Leading-Color Two-Loop QCD Corrections for Three-Photon Production at Hadron Colliders, JHEP 01 (2021) 078 [arXiv:2010.15834] [INSPIRE].

[79] L. Chen, A prescription for projectors to compute helicity amplitudes in D dimensions, Eur. Phys. J. C 81 (2021) 417 [arXiv: 1904.00705] [INSPIRE].

[80] T. Peraro and L. Tancredi, Physical projectors for multi-leg helicity amplitudes, JHEP 07 (2019) 114 [arXiv: 1906. 03298] [INSPIRE].

[81] T. Peraro and L. Tancredi, Tensor decomposition for bosonic and fermionic scattering amplitudes, Phys. Rev. D 103 (2021) 054042 [arXiv:2012.00820] [INSPIRE].

[82] T. Ahmed, A.H. Ajjath, L. Chen, P.K. Dhani, P. Mukherjee and V. Ravindran, Polarised Amplitudes and Soft-Virtual Cross Sections for $b \bar{b} \rightarrow Z H$ at NNLO in QCD, JHEP 01 (2020) 030 [arXiv: 1910.06347] [INSPIRE].

[83] T. Ahmed, W. Bernreuther, L. Chen and M. Czakon, Polarized $q \bar{q} \rightarrow Z+$ Higgs amplitudes at two loops in QCD: the interplay between vector and axial vector form factors and a pitfall in applying a non-anticommuting $\gamma_{5}$, JHEP 07 (2020) 159 [arXiv:2004.13753] [INSPIRE].

[84] B. Ruijl, T. Ueda and J. Vermaseren, FORM version 4.2, arXiv:1707.06453 [INSPIRE].

[85] J. Klappert, S.Y. Klein and F. Lange, Interpolation of dense and sparse rational functions and other improvements in FireFly, Comput. Phys. Commun. 264 (2021) 107968 [arXiv: 2004.01463] [INSPIRE]. 